\title{
Early trauma: long lasting, difficult to treat and transmitted to the next generation
}

\author{
Kerstin Konrad $^{1,2} \cdot$ Sabine C. Herpertz ${ }^{3}$ - Beate Herpertz-Dahlmann ${ }^{4}$
}

Published online: 13 August 2016

(C) Springer-Verlag Wien 2016

Since the early roots of psychodynamic theories at the beginning of the last century, a "happy" childhood has been regarded as fundamental to healthy human development. However, it was only recently that we discovered the devastating neurobiological scars and long-lasting impact of childhood adversity.

Childhood adversity and trauma involve various forms of extreme stress exposure induced by early physical abuse, emotional maltreatment, neglect, sexual abuse, or witnessing of violence. Early adversity affects up to $40 \%$ of children raised in the western countries, with even higher numbers in war zones or developmental countries (Scher et al. 2004). Very recently, this situation has been found to be even exacerbated by the increasing numbers of people being in flight, resulting in 21.3 million refugees, over half of whom are under the age of 18 and who are at heightened risk for traumatization (UNHCR 2015).

This is the introductory Editorial for the Special Issue on the "Neurobiology of early adversity and trauma".

Beate Herpertz-Dahlmann

bherpertz@ukaachen.de

1 Child Neuropsychology Section, Department of Child and Adolescent Psychiatry, Psychosomatics and Psychotherapy, University Hospital of the RWTH Aachen, Aachen, Germany

2 JARA-BRAIN Institute Molecular Neuroscience and Neuroimaging, Forschungszentrum Jülich GmbH and RWTH Aachen University, Jülich, Germany

3 Department of General Psychiatry, Center for Psychosocial Medicine, University of Heidelberg, Heidelberg, Germany

4 Department of Child and Adolescent Psychiatry, Psychosomatics and Psychotherapy, University Hospital of the RWTH Aachen, Aachen, Germany
Thorough evidence suggests that early adversity and childhood trauma have dramatic and long-lasting effects on neurobiological systems and functions that are involved in different forms of psychopathology as well as in health in general, including not only psychiatric diseases and stress responses, but also cognitive functions as well as reproductive performance, and risk for infectious and cardiometabolic diseases later in life (Gluckman et al. 2005). The associated direct and indirect costs of childhood adversity to society are enormous, consuming a substantial percentage of current state and federal budgets (Shonkoff 2012). These include health care expenditures paying for the consequences of unhealthy lifestyles (e.g., obesity, tobacco/alcohol/substance abuse) and costs of chronic diseases that may have their origins early in life. In addition, it has been demonstrated that psychiatric patients who have suffered from early traumatization are more difficult to treat than patients with a "healthy" childhood and are at greater risk for higher symptom severity and comorbidities, chronic course and poorer social functioning and quality of life (Teicher and Samson 2013).

Recently, these findings have also influenced the classification of psychiatric disorders within the DSM-5 (APA 2013), including a separate category of "trauma and stressrelated disorders" based on the concept of revised diagnoses along the lines of biological rather than clinical phenomenology.

However, still very little is known about the exact neurobiological mechanisms by which childhood trauma heightens vulnerability to psychopathology and diseases and vice versa which adaptive biological processes are associated with resilient phenotypes. In addition, it remains to be elucidated how neurobiological alterations induced by early trauma and adversity might be transmitted to the next generation. 
A number of large funding initiatives were initiated during the last years [e.g., National Institute of Health (NIH)] or by the German Federal Ministry of Research and Education (BMBF) to close these gaps and to improve long-term outcomes of individuals with childhood traumatization in the future. In April 2015, the MinervaGentner Symposium on "The intergenerational transmission of childhood adversity" was held in Heidelberg, Germany bringing together world-leading scientists from Israel, Germany and other countries to exchange the newest methods and findings in neurobiological research in this field.

The collection of papers included in this Special Issue will provide an overview of results presented at this symposium representing a timely selection of recent findings on neurobiological research of childhood adversity and trauma. In two review papers (Bock et al.; Lebowitz et al.) and four original articles, the neurobiological consequences of childhood trauma on the individual and its potential impact on the next generation are demonstrated. Neurobiological assessments suited for application in human studies are presented including proven measures of hypothalamic-pituitary-adrenal axis (HPA axis), such as diurnal cortisol levels (Fuchs et al.; Puetz et al.), the autonomous nervous system (ANS), such as heart rate variability (HRV) (Meyer et al.) and different structural brain measures (Kelly et al.). The following core topics within the current research on the neurobiology of early adversity are addressed:

1. What are the specific neurobiological consequences of early trauma on the brain, neuroendocrine and epigenetic level? How much are they dependent on the type, timing and duration of adversity and influenced by gender and the genetic make-up of the individual?

Here, animal models might enable a more systematic characterization of the spectra of neurobiological alterations induced by early trauma in a way that is impossible in human studies. The identification and characterization of sensitive periods in which environmental experience have the strongest impact on neural trajectories will be essential for our understanding of the consequences of trauma on mental health. In a comprehensive review, Bock et al. present work from animal and human studies to demonstrate that earlylife traumas can induce functional 'scars' in the brain, especially in brain circuits, which are essential for emotional control, learning and memory functions. The aspect of "biological scars" is also addressed by Kelly et al. investigating the sexually dimorphic impact of maltreatment on brain structures in children and adolescents including measures of cortical thickness, surface area and gyrification. Their findings point to deviant patterns of brain structures within a broad network of emotion regulation and theory-of-mind associated with maltreatment with only very few differences between the sexes.

2. What are the neurobiological mechanisms underlying the intergenerational cycle of maltreatment? Again, important questions involve the timing and severity of the traumatic events (including developmental programming in the prenatal phase, early postnatal effects as well as traumata occurring later in childhood).

In this context, Lebowitz et al. summarized evidence for pathways and mechanisms of the cross-generational influences on childhood anxiety disorders. The authors stress the important aspects of the prenatal environment affected by maternal anxiety, as well as the development and functioning of the oxytocinergic system, and genetic and epigenetic transmission. In addition, Bock et al. summarize the increasing evidence for transgenerational cycles of environmentally driven neuronal and behavioral adaptations mediated by epigenetic mechanisms and postulate that, dependent on type, time point and duration of adversity, also positive functional adaptations may occur in the relevant brain pathways, leading to better stress coping and resilience against adversities later in life.

One possible mechanism of transmission of adversity but also of resilience is through parenting behaviours. More recently, research has begun to explore the concept of "social and biological synchrony" within the parent-child relationship as it has been demonstrated that social synchrony (the online coordination of social behavior between parent and infant in the gaze, vocalization, affect, and touch modalities) triggers biological synchrony between the parent's and child's physiology, such as synchronized heart rhythms, oxytocin levels and brain activation patterns (Feldman 2015). This important issue is addressed within a study by Fuchs et al. investigating alterations of adrenocortical attunement in mothers with a history of childhood abuse and their 5-month-old boys and girls. The findings demonstrate that sex-specific neurobiological attunement between mother and child might compose a mechanism for the transgenerational transmission of adverse childhood experiences.

3. How can these neurobiological findings be incorporated into clinical practice with regard to diagnostics and more specific interventions?

Early adversity often increases symptom severity and comorbidity and decreases treatment response across various diagnostic categories. Puetz et al. critically 
discuss the limitations of categorical diagnoses within a group of children with early-life stress and highlight the importance of incorporating dimensional assessments and neurobiological factors into psychopathological evaluations. They demonstrate that children with early adversity showed a combined symptom profile of clinical range internalizing and externalizing problems as well as abnormal HPA functioning in the absence of trauma-related diagnoses. In addition, Meyer et al. investigated heart rate variability (HRV) as a reliable psychophysiological marker for the autonomous nervous system functioning within adult patients of Posttraumatic Stress Disorder (PTSD), current Borderline Personality Disorder (BPD) and BPD in remission as well as in healthy volunteers. Alterations in HRV were related to early-life maltreatment or associated psychological factors rather than diagnostic entities.

Such findings suggest that the identification of different neurobiological pathways to psychopathology indeed might help to determine diagnostic algorithms and treatment guidelines that differentiate between individuals with and without early traumatization and thereby potentially improve long-term prognosis of these difficult-to-treat patients.

As guest editors, we feel that this special issue illustrates the breadth of issues and opportunities within the emerging field of trauma research and hopefully will further stimulate interdisciplinary research involving child, adolescent and adult psychiatry, (epi)genetics and neuroscience. It is our hope that-if we identify the specific neurobiological pathways by which early experiences alter neurobiology-our society will also take more seriously the challenge to protect the children in our midst against early maltreatment.

We would like to thank all contributors for their highquality papers and the Editor-in-Chief of the Journal of Neural Transmission, Professor Dr. Peter Riederer, and Dr. Christian Riederer for his ongoing support as well as the Springer Verlag, for affording us the chance to guest edit this special issue.

\section{References}

American Psychiatric Association (2013) Diagnostic and statistical manual of mental disorders, 5th edn. American Psychiatric Association, Washington, DC

Feldman R (2015) Sensitive periods in human social development: new insights from research on oxytocin, synchrony, and highrisk parenting. Dev Psychopathol 27(2):369-395

Gluckman PD, Hanson MA, Pinal C (2005) The developmental origins of adult disease. Matern Child Nutr 1:130-141

Scher CD, Forde DR, McQuaid JR, Stein MB (2004) Prevalence and demographic correlates of childhood maltreatment in an adult community sample. Child Abuse Negl 28:167-180

Shonkoff JP (2012) Leveraging the biology of adversity to address the roots of disparities in health and development. Proc Natl Acad Sci USA 109(Suppl 2):17302-17307

Teicher MH, Samson JA (2013) Childhood maltreatment and psychopathology: a case for ecophenotypic variants as clinically and neurobiologically distinct subtypes. Am J Psychiatry 170(10):1114-1133

The UN Refugee Agency (2015) Global trends 2015. http://www. unhcr.org/global-trends-2015.html 